\title{
Dronedarone: evidence supporting its therapeutic use in the treatment of atrial fibrillation
}

\section{Renee M Sullivan \\ Brian Olshansky}

Division of Cardiovascular Medicine, University of lowa Hospitals and Clinics, lowa City, lowa, USA
Correspondence: Brian Olshansky Cardiovascular Division, 200 Hawkins Dr, 4426a JCP, University of lowa Hospitals and Clinics, lowa City, IA 52242, USA

Tel + I 31 9-356-2344

Email brian-olshansky@uiowa.edu
This article was published in the following Dove Press journal:

Core Evidence

14 September 2010

Number of times this article has been viewed

Introduction: Dronedarone, a benzofuran derivative with a structure similar to amiodarone, has been developed as a potential therapy for patients with atrial fibrillation.

Aim: To review the published evidence regarding the efficacy and safety of dronedarone use in patients with atrial fibrillation.

Evidence review: Available evidence suggests that dronedarone $400 \mathrm{mg}$ orally twice daily can lengthen the time to and decrease the overall recurrence of atrial fibrillation compared with placebo. Dronedarone may reduce risk of mortality and cardiovascular hospitalization. Patients with atrial fibrillation receiving dronedarone had improved ventricular rate control compared with patients receiving placebo. Dronedarone is associated with few serious adverse events except, notably, in patients with decompensated heart failure.

Place in therapy: Dronedarone may have a role in rate and rhythm control for patients with atrial fibrillation. Dronedarone can reduce unique, but potentially serious, end points in patients with atrial fibrillation. Despite this, the exact role of dronedarone in the management of patients with atrial fibrillation continues to emerge. It remains uncertain if dronedarone should be considered a primary treatment strategy for atrial fibrillation. Dronedarone should not be administered to patients with decompensated heart failure.

Conclusion: Dronedarone is a unique drug that may serve a key role to treat patients with atrial fibrillation.

Keywords: dronedarone, atrial fibrillation

\section{Core evidence clinical impact summary for dronedarone}

\begin{tabular}{|lll|}
\hline $\begin{array}{l}\text { Outcome } \\
\text { measure }\end{array}$ & Evidence & Implications \\
\hline $\begin{array}{l}\text { Disease-oriented } \\
\text { evidence }\end{array}$ & DAFNE & $\begin{array}{l}\text { Use of dronedarone was associated with a longer time to atrial } \\
\text { fibrillation recurrence; likewise, patients receiving dronedarone, } \\
400 \mathrm{mg} \text { orally twice daily, were more likely to maintain sinus } \\
\text { rhythm compared with patients receiving placebo }\end{array}$ \\
& EURIDIS and & $\begin{array}{l}\text { Dronedarone } 400 \mathrm{mg} \text { orally twice daily lengthened the time to } \\
\text { atrial fibrillation recurrence, as well as symptoms associated } \\
\text { with atrial fibrillation, compared with placebo }\end{array}$ \\
& DIONYSOS & $\begin{array}{l}\text { More patients on dronedarone had atrial fibrillation recurrence } \\
\text { or stopped the drug due to intolerance or lack of efficacy } \\
\text { compared with patients receiving amiodarone } \\
\text { ERATO }\end{array}$ \\
& $\begin{array}{l}\text { Dronedarone use was associated with decrease in ventricular } \\
\text { rate, both at rest and with exercise }\end{array}$ \\
&
\end{tabular}




\begin{tabular}{|lll|}
\hline $\begin{array}{l}\text { (Continued) } \\
\text { Outcome } \\
\text { measure }\end{array}$ & Evidence & Implications \\
\hline & ATHENA & $\begin{array}{l}\text { The use of dronedarone was associated with decreased } \\
\text { cardiovascular deaths and arrhythmic deaths compared with } \\
\text { placebo. There was also a decrease in hospitalizations for atrial } \\
\text { fibrillation and acute coronary syndrome in patients receiving } \\
\text { dronedarone compared with placebo } \\
\end{array}$ \\
& ANDROMEDA & $\begin{array}{l}\text { Dronedarone } 400 \text { mg orally twice daily was associated with an } \\
\text { acute increase in death in patients with new or worsening New } \\
\text { York Heart Association functional class III or IV heart failure }\end{array}$ \\
$\begin{array}{l}\text { Patient-oriented } \\
\text { evidence }\end{array}$ & & \\
$\begin{array}{l}\text { Economic } \\
\text { evidence }\end{array}$ & None & \\
\hline
\end{tabular}

\section{Introduction}

Pharmacologic management is the primary means by which patients with atrial fibrillation are treated. Results of large, randomized, controlled clinical trials have demonstrated the influence of rate control and rhythm control strategies ${ }^{1,2}$ on important measurable outcomes but, despite the wealth of data, a universal treatment approach does not exist. Individualized management strategies, based on a composite of studies weighing the risks and benefits of rate control, rhythm control, and anticoagulation, are required to best treat each patient with this atrial fibrillation. In addition, catheter ablation is gaining approval in the management of specific patient group who have atrial fibrillation.

Antiarrhythmic drug therapy that theoretically may aid in maintaining sinus rhythm and thereby improve a host of clinical end points is considered by some to be a key component in managing patients with atrial fibrillation. To date, however, clinical trials have not demonstrated any specific value of an antiarrhythmic drug strategy on hard end points such as mortality and hospitalization. Antiarrhythmic drugs may have inherent risks that outweigh their benefits regarding these end points, but perhaps the real reasons to treat atrial fibrillation still needs to be better clarified.

Recently, as a result of several controlled clinical trials, a novel antiarrhythmic drug, dronedarone, has gained approval for use in patients with atrial fibrillation. The data, however, raise questions regarding the philosophy behind treating patients with atrial fibrillation, particularly regarding the use of an antiarrhythmic drug. This article addresses the role of dronedarone in the management of atrial fibrillation and shows how dronedarone may potentially augment our ability to better affect clinical outcomes in patients with this condition.

\section{Why treat atrial fibrillation?}

Reduction in symptoms, enhanced ability to perform activities of daily living, and overall improved quality of life are critical issues that form the basis of present day therapy for patients with atrial fibrillation. These issues receive little attention in clinical studies. Mortality has been studied as an end point in several large trials and, no doubt, atrial fibrillation has been associated with greater risk of death..$^{3-5}$

Some studies simply look at time to first atrial fibrillation recurrence, whereas others define atrial fibrillation burden. Many episodes of atrial fibrillation, however, go unrecognized, are of brief duration, or have little import. Although these measures may help discern the antiarrhythmic efficacy of a drug, they provide little information about how to treat the underlying problem. Rate and rhythm control strategies have been tested and compared, but these do not address a comprehensive approach to the management of patients with atrial fibrillation since rarely is either strategy the lone approach. In fact, we have learned that neither approach is necessarily superior with regard to risk of stroke, survival, or any important clinical end point. ${ }^{1,2,6}$ Irrespective of rate and rhythm controls, recent data support a unique role for dronedarone to treat patients with atrial fibrillation. ${ }^{7}$

\section{Dronedarone - pharmacologic and electrophysiologic properties}

Current antiarrhythmic drug strategies to help manage atrial fibrillation and maintain sinus rhythm tend to focus on the use of amiodarone, among other drugs. Amiodarone, however, is not approved for this purpose in the United States. Although perhaps dronedarone is the most efficacious drug to maintain sinus rhythm and control ventricular rate should atrial fibrillation recur, its use comes with the risk of adverse 
effects that make the drug untenable to many treating physicians and patients alike.

Dronedarone is structurally similar to amiodarone but lacks an iodine moiety. As such, it holds some properties akin to amiodarone, but has others that make dronedarone unique. Based on the Vaughan-Williams classification, ${ }^{8}$ dronedarone, similar to amiodarone, is considered a class III antiarrhythmic drug. Dronedarone can decrease sinus rate, increase Wenckebach cycle length, the AH interval, atrial, atrioventricular node, and ventricular effective refractory periods, ${ }^{9}$ as well as inhibit $\beta$-adrenoreceptor stimulation, ${ }^{10,11}$ at least in canine models. Rat and pig models of coronary ischemia have also demonstrated dronedarone to reduce ventricular fibrillation episodes at low doses and eliminate ventricular fibrillation and mortality at higher doses, ${ }^{12}$ as well as reduce ventricular tachycardia and premature ventricular contractions. ${ }^{13}$ Upon reperfusion, dronedarone reduced mortality at low doses and eliminated ventricular fibrillation or mortality at higher doses. ${ }^{12}$ In comparison, during ischemia, high doses of amiodarone were shown to reduce the incidence of mortality ${ }^{12}$ and ventricular fibrillation (although not to a significant degree), ventricular tachycardia, and premature ventricular contractions. ${ }^{13}$

Dronedarone has demonstrated an inhibitory effect on the following potassium channels: rapid delayed rectifier, slow delayed rectifier, acetylcholine-activated, inward rectifier, and sustained currents. ${ }^{14-16}$ There was reduction of inward currents of the rapid sodium channel and L-type and T-type calcium currents. ${ }^{8}$ In addition, suppression of automaticity of the sinoatrial node due to prolongation of action potential duration and change in slope of phase 4 depolarization has been observed. ${ }^{17} \alpha$ - and $\beta$-adrenoreceptor antagonism have been seen. ${ }^{10,18}$

The electrophysiologic properties of dronedarone during chronic administration may differ from those of amiodarone. In a patch clamp study of canine Purkinje fibers, papillary muscle, and ventricular myocytes, QT prolongation and lengthening of the action potential duration, seen with amiodarone, were not observed with dronedarone. ${ }^{19}$ This difference may be related to lack of accumulation of metabolites with dronedarone as opposed to the effects of the parent drug. Alternatively, a rabbit model evaluating the long-term use of these drugs showed both increased action potential duration and effective refractory period..$^{20}$

Although dronedarone is devoid of many of the concerning side effects associated with amiodarone, it may also lack the antiarrhythmic potency of amiodarone. Compared with amiodarone, dronedarone is less lipophilic, has less tissue accumulation, and has a shorter elimination half-life (27-31 hours). The drug is eliminated mainly through the GI tract, as opposed to the hepatic metabolism of amiodarone. Dronedarone requires twice daily dosing. There is no need for an aggressive loading regimen, and steady state is reached within 4-8 days of initiation. ${ }^{8} \mathrm{~N}$-debutyldronedarone, the major metabolite of dronedarone, exhibits similar characteristics to dronedarone's antiarrhythmic drug effect. Dronedarone has few drug-drug interactions and is rarely proarrhythmic. As such it can be initiated without need for hospitalization in properly selected patients.

Dronedarone is a moderate inhibitor of CYP3A4 and a weak inhibitor of CYP2D6, and thereby impacts the metabolism of some drugs, such as statins, calcium-channel antagonists, $\beta$-blockers, and digoxin. ${ }^{8}$ There can be drugs that affect metabolism (such as tacrolimus). Although dronedarone can increase creatinine, it does not affect the glomerular filtration rate. ${ }^{8}$

\section{Controlled clinical trials showing antiarrhythmic efficacy of dronedarone Rhythm control Dronedarone Atrial Fibrillation study after Electrical Cardioversion}

The Dronedarone Atrial Fibrillation study after Electrical Cardioversion (DAFNE) was a mutlticountry, double-blind, placebo-controlled study that determined the proper dose of dronedarone to prevent recurring atrial fibrillation after cardioversion. ${ }^{21}$ Patients with persistent atrial fibrillation of 72 hours to 12 months of duration anticoagulated with warfarin for at least 3 weeks were randomized to one of the following: dronedarone (400, 600, or $800 \mathrm{mg}$ twice daily) or placebo. Patients with more than 2 cardioversions in the last 6 months, those with a reversible cause of atrial fibrillation, recent myocardial ischemia, QT interval $>500 \mathrm{~ms}$ or history of torsades de pointes, New York Heart Association (NYHA) Functional Class III or IV heart failure, left ventricular ejection fraction $<35 \%$, or those with an implantable cardioverter defibrillator were excluded from the study. Electrical cardioversion was performed if patients remained in atrial fibrillation 5-7 days after starting therapy; the therapy continued for 6 months if patients were successfully cardioverted.

One hundred ninety-nine patients in whom sinus rhythm was restored were followed with transtelephonic electrocardiogram (ECG) monitoring throughout the trial. The median 
time to atrial fibrillation recurrence of at least 10-minute duration was 5.3 days in the placebo group and 60 days in the dronedarone $400 \mathrm{mg}$ twice daily group (relative risk reduction, 55\%; 95\% confidence interval [CI], 72-28; $P=0.001)$. There were no significant differences in the other groups. Thirty-five percent of those taking dronedarone $400 \mathrm{mg}$ twice daily maintained sinus rhythm at 6 months vs $10 \%$ in the placebo group. There was greater incidence of conversion to sinus rhythm in the dronedarone groups (5.8\% with $400 \mathrm{mg}$ twice daily, 8.2\% with $600 \mathrm{mg}$ twice daily, and $14.8 \%$ with $800 \mathrm{mg}$ twice daily) compared with $3.1 \%$ with placebo $(P=0.026)$, but there was no difference in the success of electrical cardioversion between the groups. Although more efficacious, patients in the dronedarone groups were more likely to discontinue therapy $(3.9 \%, 400$ mg twice daily; 7.6\%, $600 \mathrm{mg}$ twice daily; and 22.6\%, 800 mg twice daily) compared with placebo $(0 \%)$. This was usually due to gastrointestinal complaints, specifically, diarrhea.

Several important issues regarding the use of dronedarone emerged from this study. Oral administration of dronedarone $400 \mathrm{mg}$ twice daily could safely maintain sinus rhythm in patients with previous atrial fibrillation even if only a small percent of patients actually responded to the drug compared with placebo based on the criteria of 10-minute durations of atrial fibrillation. Side effects were dose dependent, and although tolerable and not as severe as those experienced with some other antiarrhythmic drugs, they may limit the benefits of the drug regarding symptom reduction. This is the reason to limit dronedarone dosing to $400 \mathrm{mg}$ twice daily. From this point, no other doses of dronedarone have been used in clinical trials.

\section{EURIDIS and ADONIS}

The European Trial in Atrial Fibrillation or Flutter Patients Receiving Dronedarone for the Maintenance of Sinus Rhythm (EURIDIS) and the American-Australian-African Trial with Dronedarone in Atrial Fibrillation or Flutter Patients for the Maintenance of Sinus Rhythm (ADONIS) were multicenter, double-blind trials that sought to determine if dronedarone was superior to placebo in maintaining sinus rhythm after conversion from atrial flutter or fibrillation. ${ }^{22}$ These trials were sponsored by Sanofi-Aventis. They also managed and analyzed the data.

Enrolled patients had at least one episode of atrial fibrillation in the prior 3 months and were in sinus rhythm for at least 1 hour before randomization. Participants in the study had a mean age of 63 years and were mostly men. More than 50\% had hypertension. Patients were randomized 2:1 to dronedarone $400 \mathrm{mg}$ twice daily or placebo in addition to concomitant therapy, including $\beta$-blockers, calcium-channel antagonists, and digoxin. Patients with permanent atrial fibrillation, women of child-bearing age not using birth control, patients with a history of torsades de pointes, persistent heart rate $<50$ beats per minute, a PR interval $\geq 0.28 \mathrm{~s}$, second degree or more advanced heart block, those taking class I or III antiarrhythmic drugs, NYHA class III or IV heart failure or creatinine $>1.7 \mathrm{mg} / \mathrm{dL}$ were excluded.

EURIDIS randomized 411 patients to dronedarone and 201 patients to placebo. The median time to recurrence of atrial fibrillation lasting at least 10 minutes was 96 days in the dronedarone group vs 41 days in the placebo group; $37 \%$ of patients in the dronedarone group and $48 \%$ in the placebo arm were symptomatic $(P=0.006)$. At 1 year, $67 \%$ of patients on dronedarone had a recurrence compared with $78 \%$ receiving placebo (hazard ratio $[\mathrm{HR}]=0.78 ; 95 \% \mathrm{CI}, 0.64-0.96$; $P=0.01)$. Post hoc analysis showed more patients in the placebo arm had been hospitalized or died at 12 months compared with dronedarone $(32 \%$ vs $21 \%$, HR $=0.66 ; 95 \%$ CI, 0.47-0.93; $P=0.02$ ).

ADONIS randomized 417 patients to dronedarone and 208 patients to placebo. The median time to recurrence was 158 days in the dronedarone group compared with 59 days in the placebo group; $38 \%$ of patients in the dronedarone group and $45 \%$ in the placebo group were symptomatic $(P=0.02)$. At 1 year, $61 \%$ of patients on dronedarone had recurrence compared with $73 \%$ receiving placebo $(\mathrm{HR}=0.73 ; 95 \% \mathrm{CI}, 0.59-0.89 ; P=0.002)$. Post hoc analysis showed no difference between the groups when comparing hospitalizations or death at 12 months $(P=0.22)$.

Although EURIDIS and ADONIS showed that dronedarone can lengthen the time to first recurrence of atrial fibrillation, these data did not indicate that most patients taking dronedarone remained arrhythmia free; a substantial number of participants in the study had recurrence at 1 year. In addition, all recurrent episodes may not have been detected, particularly if patients were asymptomatic and did not transmit an ECG transtelephonically. Furthermore, time to the first recurrence did not necessarily indicate an improvement in frequency or duration of atrial fibrillation episodes. These data do not indicate a strong antiarrhythmic effect of dronedarone, and these data cannot be compared with the antiarrhythmic drug efficacy of any other drug as this population is unique. 


\section{DIONYSOS}

The Randomized, Double-Blind Trial to Evaluate the Efficacy and Safety of Dronedarone (400 mg twice daily) vs amiodarone (600 mg everyday for 28 days, then $200 \mathrm{mg}$ everyday thereafter) for at least 6 months for the maintenance of sinus rhythm in patients with atrial fibrillation (DIONYSOS) study sought to directly compare the risks and benefits of dronedarone and amiodarone by measuring efficacy with 12 lead ECGs to document atrial fibrillation recurrence and safety of the 2 drugs. ${ }^{23}$

A total of 504 patients with atrial fibrillation for at least 72 hours were randomized to dronedarone $400 \mathrm{mg}$ twice daily ( $\mathrm{N}=249$ ) or amiodarone $600 \mathrm{mg}$ daily for 28 days, and then $200 \mathrm{mg}$ thereafter $(\mathrm{N}=255)$. Patients were excluded if they had received amiodarone previously or had contraindications to its use, as well as if they had severe congestive heart failure (including those with NYHA class III or IV symptoms) or high-degree atrioventricular block. Patients who did not achieve sinus rhythm after initiation of the study drug underwent electrical cardioversion. Patients who failed electrical cardioversion or failed spontaneous conversion after drug initiation were considered to have atrial fibrillation recurrence in the analysis. Most participants in the study were males, had persistent atrial fibrillation, had hypertension, and were receiving oral anticoagulants and $\beta$-blockers at the time of enrollment.

During a mean follow up of 7 months, $75.1 \%$ of patients receiving dronedarone had either recurrence of atrial fibrillation or stopped the medication due to intolerance or lack of efficacy compared with $55.8 \%$ in the amiodarone arm $(\mathrm{HR}=1.59 ; 95 \% \mathrm{CI}, 1.28-1.98 ; P<0.0001)$. More patients had recurrent atrial fibrillation with dronedarone compared with amiodarone (63.5\% vs $42 \%)$. Premature drug discontinuation occurred with both drugs (10.4\% with dronedarone vs $13.3 \%$ with amiodarone).

Adverse event rates were high for both the drugs (39.3\% with dronedarone vs $44.5 \%$ with amiodarone; $\mathrm{HR}=0.80 ; 95 \% \mathrm{CI}, 0.60-1.07 ; P=0.13)$. However, there were fewer thyroid, neurological, skin, and eye events with dronedarone compared with amiodarone. Similar to other studies, dronedarone was associated with more adverse gastrointestinal events than amiodarone. Participants receiving dronedarone had less reduction in heart rate, had fewer QT prolongations beyond $500 \mathrm{~ms}$, and had fewer elevated international normalized ratio (INR) compared with those on amiodarone. Although dronedarone was not as efficacious as amiodarone in maintaining sinus rhythm, considering its lower risk of side effects, it may be reasonable to consider the use of dronedarone before initiating amiodarone.

Unfortunately, $75 \%$ of those randomized to dronedarone had recurrent atrial fibrillation, did not convert to sinus rhythm, or had to stop the drug due to intolerance or lack of efficacy. Even higher rates of atrial fibrillation recurrence may have been present as recurrence was dependent on intermittent 12 lead ECG recordings. On the other hand, this strategy of monitoring seems more representative of what actually occurs in clinical practice, and it is possible that short bouts of atrial fibrillation detected by transtelephonic monitoring may not be clinically relevant. Despite higher risk of side effects, amiodarone was more effective to maintain sinus rhythm. Although not reported, in long-term follow-up, amiodarone may have a greater risk for severe or potentially life-threatening adverse effects.

\section{Dronedarone vs amiodarone rhythm control - meta-analysis}

A meta-analysis comparing the efficacy and safety of dronedarone with amiodarone to prevent recurrent atrial fibrillation was recently published. ${ }^{24}$ Four dronedarone studies and 4 amiodarone studies were included for indirect comparison. One study, DIONYSOS, was included for direct comparison. Although dronedarone and amiodarone could prevent atrial fibrillation recurrence compared with placebo (odds ratio $[\mathrm{OR}]=0.79 ; 95 \% \mathrm{CI}, 0.33-1.87$ and $\mathrm{OR}=0.12 ; 95 \% \mathrm{CI}$, 0.08-0.19, respectively), when compared to each other, amiodarone was more potent $(\mathrm{OR}=0.16$; 95\% CI, 0.06-0.42). In terms of safety, dronedarone was more favorable than amiodarone, which was associated with higher odds of study drug termination $(\mathrm{OR}=6.65 ; 95 \% \mathrm{CI}, 1.13-39.3)$. These data highlight the efficacy of amiodarone as an antiarrhythmic drug and indicate that dronedarone is not as effective, at least in the populations studied.

It should be noted, however, that all meta-analyses are subject to specific weaknesses of study design and end-point monitoring. As we discussed earlier, time to recurrence of atrial fibrillation may not be the most appropriate end point to consider. Depending on how the data are analyzed and the specific end point selected, dronedarone may or may not appear to be a more successful drug in the management of atrial fibrillation. Clinically, it is hard to incorporate sophisticated statistical manipulations to better understand efficacy. In reality, if a patient has recurrent symptomatic atrial fibrillation, even if it occurs several months or even years later, it may be interpreted by the clinician and the patient as a failure of the drug. In selected patients, maintenance of 
sinus rhythm may have value and may be best achieved by an ablation strategy. When combining clinical studies that evaluate antiarrhythmic drugs that have different statistical methodologies and end points, it can be quite difficult to draw meaningful conclusions. It stated that dronedarone may have antiarrhythmic efficacy to prevent atrial fibrillation recurrence, but it does not appear to be as effective as amiodarone and, considering the side effects of the drugs, it remains uncertain whether it will establish itself as a drug that is superior to amiodarone.

\section{Rate control}

The efficacy and safety of dronedarone for the control of ventricular rate during atrial fibrillation (ERATO) study was a multicenter, double-blind, placebo-controlled trial conducted to determine the efficacy of dronedarone to control ventricular response in patients with permanent atrial fibrillation. ${ }^{25}$ After 2 weeks of screening, patients with symptomatic and permanent atrial fibrillation with a ventricular rate $\geq 80$ beats per minute on a 6-second rhythm strip were randomized in a 1:1 fashion to dronedarone $400 \mathrm{mg}$ twice daily $(\mathrm{N}=85)$ or placebo $(\mathrm{N}=89)$, in addition to their usual therapy. At baseline, less than $50 \%$ of patients were taking calcium-channel antagonists or digoxin and just over $50 \%$ were taking $\beta$-blockers. Patients were excluded if they were taking other antiarrhythmic drugs, had unstable angina, a history of torsades de pointes, third-degree heart block or sinus node disease, or NYHA Functional Class III or IV heart failure.

At day 14, patients in the dronedarone arm had a ventricular rate 11 beats per minute on 24-hour Holter monitor compared with placebo $(P<0.0001)$, not accounted for by the use of other medications. When added to $\beta$-blockers or digoxin, dronedarone had a significant impact on decreasing heart rate compared with placebo $(-14.9$ vs -11.5 beats per minute, $P<0.0001$ ), but the change was not significant in conjunction with calcium-channel antagonists $(-5.1$ beats per minute, $P=0.1$ ). The control of ventricular rate with dronedarone over placebo persisted with both submaximal and maximal exercise testing at day 14 compared with day $0(P<0.0001)$. At 4 months, there continued to be control of the ventricular rate over a 24-hour period in the dronedarone arm compared with the placebo arm $(P<0.001)$. Few adverse events occurred in the study: there was one death of a patient with congenital heart disease and an abnormal EKG, which should have precluded entry into the study (EKG findings were not described) in the dronedarone arm, 3 myocardial infarctions ( 1 in the dronedarone arm, 2 in the placebo arm), 1 episode of heart failure in the dronedarone arm, and 1 case of unstable angina in the placebo arm.

Although not their primary end points, other dronedarone trials showed the value of the drug to control ventricular rate if atrial fibrillation were to recur. In DAFNE, patients in the dronedarone groups had a significant reduction in ventricular rate response at the time of recurrent atrial fibrillation compared with placebo $(P=0.0001) .{ }^{21}$ Similarly, both EURIDIS and ADONIS showed similar benefit in patients with recurrent atrial fibrillation receiving dronedarone and placebo (ventricular rate $102 \pm 25$ beats per minute in the dronedarone group and $118 \pm 29$ beats per minute in the placebo group in EURODIS, $P<0.001) ; 105 \pm 27$ beats per minute in the dronedarone group and $117 \pm 32$ beats per minute in the placebo group in ADONIS, $P<0.001 .^{22}$

These data suggest that dronedarone may have a beneficial effect for patients if atrial fibrillation were to recur. The mechanism by which dronedarone controls the ventricular response rate in atrial fibrillation remains uncertain, but nevertheless, part of symptom reduction in patients who report improvement when taking dronedarone could be, in part, due to this effect alone. The mechanism by which dronedarone affects ventricular rate may be by alteration in atrial fibrillation cycle length or by its effect on AV conduction. Important to realize, however, is that the patients enrolled in ERATO did not have a rapid ventricular response to begin with: patients in placebo had a heart rate of approximately 90 beats per minute and those randomized to dronedarone started with a heart rate of approximately 85 beats per minute. Further, the proper ventricular rate in atrial fibrillation remains uncertain. ${ }^{1,26-28}$ Nevertheless, these data suggest that dronedarone may be a drug that could be used individually rather than in combination with other rate-controlling medications, such as calcium-channel antagonists or $\beta$-adrenergic blockers. Alternatively, patients who tend to be candidates for dronedarone may require one of these medications in any event, at least for treatment of comorbid conditions such as hypertension or coronary artery disease.

\section{Novel end points of dronedarone}

A Placebo-Controlled, Double-Blind, Parallel Arm Trial to Assess the Efficacy of Dronedarone $400 \mathrm{mg}$ bid for the Prevention of Cardiovascular Hospitalization or Death from Any Cause in Patients with Atrial Fibrillation/Atrial Flutter (ATHENA) was conducted to determine if dronedarone could 
decrease the composite end point of cardiovascular hospitalizations or death in patients with atrial fibrillation. ${ }^{7}$ The trial was sponsored by Sanofi-Aventis, who also collected, managed, and analyzed the data. It is the pivotal trial that ultimately helped the drug gain approval for use in the United States. ATHENA was as large as the Atrial Fibrillation Follow-up Investigation of Rhythm Management (AFFIRM) trial and was one of the largest trials that studied the use of an antiarrhythmic drug to treat atrial fibrillation.

Patients with paroxysmal or persistent atrial fibrillation or flutter were included in the study if they were at least 70 years old or had other comorbid conditions, such as hypertension, diabetes, stroke or transient ischemic attack, or left ventricular ejection fraction $\leq 40 \%$. Dronedarone therapy was initiated in 2,301 patients and placebo was initiated in 2,327; median follow-up was $21 \pm 5$ months. Almost all patients ( $n=4,544)$ had left ventricular function evaluation; only $4 \%$ had a left ventricular ejection fraction $<35 \%$ and $12 \%$ had a left ventricular ejection fraction $<45 \%$. Most patients were taking a $\beta$-blocker, an angiotensin-converting enzyme inhibitor or an angiotensin receptor blocker therapy at the time of enrollment.

Of those randomized to dronedarone, 675 were hospitalized and 59 died; of those who received placebo, 859 had a cardiovascular event requiring hospitalization and 58 died $(\mathrm{HR}=0.76 ; 95 \% \mathrm{CI}, 0.69-0.84 ; P<0.001)$. Cardiovascular deaths were fewer in the dronedarone arm compared with placebo $(\mathrm{HR}=0.71 ; 95 \% \mathrm{CI}, 0.51-0.98$; $P=0.03)$, as were deaths from arrhythmia (HR $=0.55 ; 95 \%$ CI, 0.34-0.88; $P=0.01)$. There were fewer hospitalizations for atrial fibrillation and acute coronary syndrome in the dronedarone group compared with placebo $(P<0.001$ and $P=0.03$, respectively). In the ATHENA trial, there were more treatment-emergent adverse events in the dronedarone group compared with placebo $(P=0.05)$, including cardiac events, QT-interval prolongation, gastrointestinal events, rash, and increase in serum creatinine (all $P<0.05$ ), which led to premature discontinuation of the study drug more with dronedarone than placebo $(P<0.001)$.

A post hoc analysis of the ATHENA data determined the impact of dronedarone on stroke. ${ }^{29}$ The baseline risk of stroke was based upon the $\mathrm{CHADS}_{2}$ score for each participant (mean score of 2 in the placebo and dronedarone groups), and the occurrence of stroke was determined by review of hospitalization and death records. Only $60 \%$ of patients were receiving oral anticoagulation at the time of enrollment, and this remained steady during follow-up. Participants in both arms were considered to have a therapeutic INR of $2-3$ no more than $50 \%$ of the time; the anticoagulation status at the time of an event was not reported. There were fewer total strokes and strokes or transient ischemic attacks combined in the group receiving dronedarone compared with placebo ( $P=0.03$ for both). When the combined end points of stroke, acute coronary syndrome, and death or cardiovascular death were studied, favorable results were also seen with dronedarone compared with placebo $(P=0.002$ and $P<0.001$, respectively).

ATHENA was a large, multicenter trial that indicated that a unique and important clinical end point could be achieved with dronedarone $400 \mathrm{mg}$ twice daily. It is important to recognize that none of these patients had acute exacerbations of congestive heart failure when the drug was initiated. Furthermore, this study was unique as an antiarrhythmic drug study for atrial fibrillation, in that it did not consider the recurrence rates of atrial fibrillation or even rate control as primary objectives of the study. The combined clinical end point, while clinically important, may have little impact on the utility of this drug as the key purpose of treating patients with atrial fibrillation is to improve symptoms, reduce the risk of stroke, and improve quality of life. However, dronedarone did reduce hospitalizations for atrial fibrillation, and this alone may improve patients' quality of life. From an economic standpoint, this may also be a useful drug given the epidemic of atrial fibrillation. On the other hand, this information is quite noteworthy because no other antiarrhythmic drug to date has shown any favorable impact on mortality or hospitalization due to cardiovascular causes. In this sense, dronedarone is potentially attractive for patients with atrial fibrillation as it may affect several important clinical outcomes.

In ATHENA, dronedarone was used in a specific population of elderly patients who had modest $\mathrm{CHADS}_{2}$ scores. Although there was a reduction in stroke and acute coronary syndromes in the dronedarone arm, it should also be noted that not all patients received oral anticoagulation during the study and it is not clear if patients were adequately anticoagulated at the time of a clinical event. Similarly, it is unclear the percentage of participants who maintained sinus rhythm in ATHENA as this was not a study end point. It is possible that patients who maintained sinus rhythm were less likely to have a stroke.

Based on the results of the ATHENA trial, dronedarone appears to be relatively safe despite the greater premature discontinuation of the drug compared to placebo. The risk of major side effects that occur or can occur with amiodarone, including thyroid disease, liver toxicity, pul- 
monary toxicity, and neurological toxicity did not occur with dronedarone. In fact, compared with placebo, dronedarone had no substantial serious adverse side effects. That stated, there are potential side effects that can occur with dronedarone. These include gastrointestinal intolerance, shortness of breath, and elevation in creatinine which is not associated with renal dysfunction and may be of no clinical significance.

\section{Controlled clinical trials showing potential risks of dronedarone}

The Antiarrhythmic Trial with Dronedarone in Moderate to Severe CHF Evaluating Morbidity Decrease (ANDROMEDA) was a multicenter, double-blind study that sought to determine if the use of dronedarone $400 \mathrm{mg}$ orally twice daily could reduce the rate of heart failure hospitalizations, as well as mortality, by reducing deaths due to arrhythmia in 627 patients hospitalized with new or worsening NYHA Functional Class III or IV heart failure with a wall motion index of $\leq 1.2$, the equivalent of a left ventricular ejection fraction of $35 \% .{ }^{30}$ Among other criteria, patients were excluded if they had an acute myocardial infarction, bradycardia, or were receiving class I or III antiarrhythmic drugs. Only a quarter of the patients had atrial fibrillation at the time of randomization; $40 \%$ reported a history of atrial fibrillation.

The study was stopped early (median follow-up of 2 months) as 25 of the patients randomized to dronedarone $(\mathrm{n}=310)$ died, whereas only 12 patients randomized to placebo $(\mathrm{n}=317)$ did $(\mathrm{HR}=2.13 ; 95 \% \mathrm{CI}, 1.07-4.25 ; P=0.03)$. There was no difference in deaths after an additional 6 months of follow-up: 42 patients on dronedarone and 39 patients on placebo had died $(\mathrm{HR}=1.13 ; 95 \% \mathrm{CI}, 0.73-1.74 ; P=0.6)$. There was no difference in the primary end point of all-cause mortality or hospitalization for heart failure between the 2 groups with 53 events in participants receiving dronedarone and 40 events in the placebo group at 2 months $(\mathrm{HR}=1.38 ; 95 \% \mathrm{CI}, 0.92-2.09 ; P=0.12)$ and 74 events in the dronedarone arm and 72 events in the placebo arm after an additional 6-month follow-up $(\mathrm{HR}=1.09$; 95\% CI, $0.79-1.51 ; P=0.6)$. More patients in the dronedarone arm were hospitalized for cardiovascular reasons compared with patients receiving placebo $(P=0.02)$. Based upon the results of this trial, dronedarone carries a boxed warning advising against the use of it in patients with NYHA Functional Class IV heart failure or NYHA Functional Class II and III heart failure with recent decompensation requiring hospitalization or referral to a heart failure clinic.
There are several important issues that must be recognized regarding this trial. As mentioned above, not all patients had atrial fibrillation, and thus, this was a trial that assessed the safety of dronedarone in patients with recent onset congestive heart failure and impaired left ventricular function. In sharp contrast to the ATHENA trial in which patients could have heart failure symptoms but were considered stable, patients in ANDROMEDA were acutely decompensated. In fact, in the ATHENA trial, patients with left ventricular ejection fractions $<35 \%$ were enrolled, reaching numbers similar to the number of participants in ANDROMEDA. In ATHENA, all patients with heart failure and low ventricular ejection fractions had atrial fibrillation in contrast to the ANDROMEDA trial. Nevertheless, these data raise red flags about the use of dronedarone in patients with congestive heart failure and impaired ventricular function. We advise against the use of dronedarone in these patients. The utility of dronedarone in patients with diastolic dysfunction and intact ejection fraction has not been directly studied to date.

\section{Dronedarone in clinical practice}

The data that have been reported thus far concerning dronedarone have been collected to better understand the effects of the drug on rate and rhythm control, hospitalizations, and mortality. Many of these studies have been performed to determine if dronedarone can be utilized for a wide range of potential indications. These indications are only beginning to be understood, and the exact role of dronedarone to treat patients with atrial fibrillation remains uncertain.

To date, there have been no changes in the American College of Cardiology/American Heart Association Guidelines in regards to the use of dronedarone in the management of patients with atrial fibrillation. ${ }^{31}$ Some investigators have attempted to recommend a role for dronedarone based on the present data. ${ }^{32}$ On the other hand, the National Institute for Health and Clinical Excellence (NICE) in England and Wales has been asked to consider the addition of dronedarone to their 2006 guidelines for atrial fibrillation management. ${ }^{33}$ The original assessment was that dronedarone should not be included in the updated guidelines as it has not shown important clinical utility in lieu of its potential costs.

The most recent preliminary appraisal consultation from the NICE committee, however, suggests dronedarone may be considered for the treatment of nonpermanent atrial fibrillation if the atrial fibrillation is not controlled 
by first-line therapy, and the patient has at least one of the following: hypertension requiring medications from at least 2 different classes; diabetes mellitus; previous transient ischemic attack, stroke, or systemic embolism; left atrial diameter of $\geq 50 \mathrm{~mm}$; left ventricular ejection fraction $\leq 40 \%$, or age $\geq 70$ years without NYHA Functional Class III or IV heart failure. ${ }^{34}$ Patients who do not meet these criteria and who are already on dronedarone will have the option to continue the drug until physicians choose to stop it.

At this time, physicians face the difficult decision of whether to initiate dronedarone based upon the results of individual controlled clinical trials that often included the use of other drugs to treat atrial fibrillation, such as $\beta$-blockers, calcium-channel antagonists, or digoxin. It is not clear that dronedarone has a role as a primary treatment strategy. Its use should be avoided in cases of decompensated NYHA Functional Class II or III heart failure, as well as NYHA Functional class IV heart failure. Patients who are receiving therapy for atrial fibrillation but still remain symptomatic and those with contraindications to amiodarone may derive benefit from dronedarone.

We consider that dronedarone has a role to treat patients with atrial fibrillation to potentially improve several hard outcomes, and improve functionality, quality of life by reducing episodes of atrial fibrillation, as well as their severity. To date, the drug has few concerning serious longterm side effects and can be used for those patients with multiple comorbidities, who have symptomatic, recurrent atrial fibrillation.

Dronedarone has advantages over other antiarrhythmic drugs that may be used in the management of atrial fibrillation. The properties of the drug allow it to be initiated as an outpatient and aggressive loading is not required. Although the drug is new to the market and cost may be prohibitive for some patients, costs are oftentimes being covered by insurance companies who consider covering the drug a small price to pay in comparison to the cost of a potential hospitalization secondary to atrial fibrillation.

\section{Future directions}

Postmarket assessment and clinical hands-on experience are key issues that will determine the fate of dronedarone in clinical practice. Initial experiences have been quite variable. Many patients have suffered recurrence of atrial fibrillation while taking dronedarone; this may be because patients who are placed on dronedarone have failed other antiarrhythmic agents. Alternatively, dronedarone may not be as good an antiarrhythmic drug as it is believed to be. Furthermore, no good data, with the exception of decrease in hospitalizations, exist to suggest that dronedarone can improve symptoms and quality of life for patients. The risks and benefits of this drug in clinical practice remain uncertain and more data are needed. As data emerge, we will have a better grasp about which patient populations can benefit the most from this drug and which have the greatest risk of side effects without gaining any substantial clinical benefit. In addition, we will begin to learn which patients already receiving therapy for atrial fibrillation, on their current regimen including antiarrhythmic agents, would benefit from switching to dronedarone.

Overall, results may be disappointing as patients and physicians realize that hospitalizations and mortality are not the end points most cared about and that reduction of symptoms and complications are the most highly regarded end points. In addition, long-term follow up may suggest that side effects and adverse effects are possible with dronedarone. Twice daily dosing of the drug may prove to decrease drug regimen adherence compared with the once daily dosing of amiodarone. Finally, the cost of dronedarone may be prohibitive for some patients so that the drug is not an option for them. Costs, however, are difficult to gauge as the price varies by location, insurance plan, and other factors.

\section{Conclusion}

Dronedarone is a novel antiarrhythmic drug that can help patients return to and maintain sinus rhythm, control ventricular response during recurrent episodes of atrial fibrillation, and reduce hospitalizations and cardiovascular mortality. These important clinical end points differ from other antiarrhythmic drugs as there have been no drugs to date, which have been able to reduce cardiovascular or total mortality in combination with hospitalization reduction. Dronedarone also has a low risk of adverse side effects and therefore appears to be relatively safe. Although it may be more expensive and more difficult to take given its twice daily dosing compared with amiodarone, for patients who are at risk for developing significant amiodarone toxicity such as those who will need the drug for a long period of time and for younger, otherwise healthy patients, this drug may have a vital role in the management of their symptoms and improvement in their long-term cardiovascular outcomes.

As we continue to learn about important clinical end points and we better understand the complexities of managing atrial fibrillation, dronedarone adds a new dimension 
to the treatment possibilities, at least for some patient populations. We look forward to the continued use of dronedarone in clinical practice to determine if it, like the mythical characters its studies are named for, can stand the test of time.

\section{Disclosures}

The authors report no conflicts of interest in this work.

\section{References}

1. Wyse DG, Waldo AL, DiMarco JP, et al. A comparison of rate control and rhythm control in patients with atrial fibrillation. $N$ Engl J Med. 2002;347(23): 1825-1833.

2. Van Gelder IC, Hagens VE, Bosker HA, et al. A comparison of rate control and rhythm control in patients with recurrent persistent atrial fibrillation. $N$ Engl J Med. 2002;347(23):1834-1840.

3. Benjamin EJ, Wolf PA, D'Agostino RB, Silbershatz H, Kannel WB, Levy D. Impact of atrial fibrillation on the risk of death: the Framingham Heart Study. Circulation. 1998;98(10):946-952.

4. Corley SD, Epstein AE, DiMarco JP, et al. Relationships between sinus rhythm, treatment, and survival in the Atrial Fibrillation Follow-Up Investigation of Rhythm Management (AFFIRM) Study. Circulation. 2004;109(12):1509-1513.

5. Pedersen OD, Brendorp B, Elming H, Pehrson S, Kober L, Torp-Pedersen C. Does conversion and prevention of atrial fibrillation enhance survival in patients with left ventricular dysfunction? Evidence from the Danish Investigations of Arrhythmia and Mortality ON Dofetilide/(DIAMOND) study. Card Electrophysiol Rev. 2003;7(3):220-224.

6. Roy D, Talajic M, Nattel S, et al. Rhythm control versus rate control for atrial fibrillation and heart failure. $N$ Engl J Med. 2008;358(25): 2667-2677.

7. Hohnloser SH, Crijns HJ, van Eickels M, et al. Effect of dronedarone on cardiovascular events in atrial fibrillation. $N$ Engl J Med. 2009;360(7): 668-678.

8. Briefing Information for the Mar 18, 2009 Meeting of the Cardiovascular and Renal Drugs Advisory Committee. 2009; Available from: http:// www.fda.gov/downloads/AdvisoryCommittees/CommitteesMeetingMaterials/Drugs/CardiovascularandRenalDrugsAdvisoryCommittee/ UCM134981.pdf. Accessed Apr 19, 2010.

9. Manning A, Thisse V, Hodeige D, Richard J, Heyndrickx JP, Chatelain P. SR 33589, a new amiodarone-like antiarrhythmic agent: electrophysiological effects in anesthetized dogs. J Cardiovasc Pharmacol. 1995;25(2):252-261.

10. Hodeige D, Heyndrickx JP, Chatelain P, Manning A. SR 33589, a new amiodarone-like antiarrhythmic agent: anti-adrenoceptor activity in anaesthetized and conscious dogs. Eur J Pharmacol. 1995;279(1):25-32.

11. Djandjighian L, Planchenault J, Finance O, Pastor G, Gautier P, Nisato D. Hemodynamic and antiadrenergic effects of dronedarone and amiodarone in animals with a healed myocardial infarction. J Cardiovasc Pharmacol. 2000;36(3):376-383.

12. Manning AS, Bruyninckx C, Ramboux J, Chatelain P. SR 33589, a new amiodarone-like agent: effect on ischemia- and reperfusion-induced arrhythmias in anesthetized rats. J Cardiovasc Pharmacol. 1995;26(3): 453-461.

13. Finance O, Manning A, Chatelain P. Effects of a new amiodarone-like agent, SR 33589, in comparison to amiodarone, D, L-sotalol, and lignocaine, on ischemia-induced ventricular arrhythmias in anesthetized pigs. J Cardiovasc Pharmacol. 1995;26(4):570-576.

14. Aimond F, Beck L, Gautier P, et al. Cellular and in vivo electrophysiological effects of dronedarone in normal and postmyocardial infarcted rats. J Pharmacol Exp Ther. 2000;292(1):415-424.
15. Altomare C, Barbuti A, Viscomi C, Baruscotti M, DiFrancesco D. Effects of dronedarone on acetylcholine-activated current in rabbit SAN cells. Br J Pharmacol. 2000;130(6):1315-1320.

16. Guillemare E, Marion A, Nisato D, Gautier P. Inhibitory effects of dronedarone on muscarinic $\mathrm{K}+$ current in guinea pig atrial cells. J Cardiovasc Pharmacol. 2000;36(6):802-805.

17. Sun W, Sarma JS, Singh BN. Electrophysiological effects of dronedarone (SR 33589), a noniodinated benzofuran derivative, in the rabbit heart: comparison with amiodarone. Circulation. 1999;100(22): 2276-2281.

18. Chatelain P, Meysmans L, Matteazzi JR, Beaufort P, Clinet M. Interaction of the antiarrhythmic agents SR 33589 and amiodarone with the beta-adrenoceptor and adenylate cyclase in rat heart. Br J Pharmacol. 1995;116(3):1949-1956.

19. Varro A, Takacs J, Nemeth M, et al. Electrophysiological effects of dronedarone (SR 33589), a noniodinated amiodarone derivative in the canine heart: comparison with amiodarone. Br J Pharmacol. 2001;133(5): 625-634.

20. Sun W, Sarma JS, Singh BN. Chronic and acute effects of dronedarone on the action potential of rabbit atrial muscle preparations: comparison with amiodarone. J Cardiovasc Pharmacol. 2002;39(5):677-684.

21. Touboul P, Brugada J, Capucci A, Crijns HJ, Edvardsson N, Hohnloser $\mathrm{SH}$. Dronedarone for prevention of atrial fibrillation: a dose-ranging study. Eur Heart J. 2003;24(16):1481-1487.

22. Singh BN, Connolly SJ, Crijns HJ, et al. Dronedarone for maintenance of sinus rhythm in atrial fibrillation or flutter. $N$ Engl J Med. 2007; 357(10):987-999.

23. LeHeuzey JY, DeFerrari GM, Radzik D, Santini M, Zhu J, Davy JM. A Short-Term, Randomized, Double-Blind, Parallel-Group Study to Evaluate the Efficacy and Safety of Dronedarone versus Amiodarone in Patients with Persistent Atrial Fibrillation: The DIONYSOS Study. J Cardiovasc Electrophysiol. In press 2010.

24. Piccini JP, Hasselblad V, Peterson ED, Washam JB, Califf RM, Kong DF. Comparative efficacy of dronedarone and amiodarone for the maintenance of sinus rhythm in patients with atrial fibrillation. $J \mathrm{Am}$ Coll Cardiol. 2009;54(12):1089-1095.

25. Davy JM, Herold M, Hoglund C, et al. Dronedarone for the control of ventricular rate in permanent atrial fibrillation: the Efficacy and safety of dRonedArone for the cOntrol of ventricular rate during atrial fibrillation (ERATO) study. Am Heart J. 2008;156(3):527, e521-e529.

26. Olshansky B, Rosenfeld LE, Warner AL, et al. The Atrial Fibrillation Follow-up Investigation of Rhythm Management (AFFIRM) study: approaches to control rate in atrial fibrillation. J Am Coll Cardiol. 2004; 43(7):1201-1208.

27. Van Gelder IC, Wyse DG, Chandler ML, et al. Does intensity of rate-control influence outcome in atrial fibrillation? An analysis of pooled data from the RACE and AFFIRM studies. Europace. 2006;8(11): 935-942.

28. Van Gelder IC, Groenveld HF, Crijns HJ, et al. Lenient versus strict rate control in patients with atrial fibrillation. N Engl J Med. 2010;362(15): 1363-1373.

29. Connolly SJ, Crijns HJ, Torp-Pedersen C, et al. Analysis of stroke in ATHENA: a placebo-controlled, double-blind, parallel-arm trial to assess the efficacy of dronedarone $400 \mathrm{mg}$ BID for the prevention of cardiovascular hospitalization or death from any cause in patients with atrial fibrillation/atrial flutter. Circulation. 2009;120(13): 1174-1180.

30. Kober L, Torp-Pedersen C, McMurray JJ, et al. Increased mortality after dronedarone therapy for severe heart failure. N Engl J Med. 2008; 358(25):2678-2687.

31. Fuster V, Ryden LE, Cannom DS, et al. ACC/AHA/ESC 2006 Guidelines for the Management of Patients with Atrial Fibrillation: a report of the American College of Cardiology/American Heart Association Task Force on Practice Guidelines and the European Society of Cardiology Committee for Practice Guidelines (Writing Committee to Revise the 2001 Guidelines for the Management of Patients With Atrial Fibrillation): developed in collaboration with the European Heart Rhythm Association and the Heart Rhythm Society. Circulation. 2006;114(7): e257-e354. 
32. Prystowsky EN, Camm J, Lip GY, et al. The Impact of New and Emerging Clinical Data on Treatment Strategies for Atrial Fibrillation. J Cardiovasc Electrophysiol. 2010.

33. The management of atrial fibrillation. NICE clinical guideline 36. 2006; Available from: http://www.nice.org.uk/nicemedia/live/10982/30052/ 30052.pdf
34. Atrial fibrillation - dronedarone: appraisal consultation document 2. Available from: http://www.nice.org.uk/guidance/index.jsp? action $=$ article $\&$ o=48099. Accessed Apr 19, 2010.
Core Evidence

\section{Publish your work in this journal}

Core Evidence is an international, peer-reviewed open-access journal evaluating the evidence underlying the potential place in therapy of drugs throughout their development lifecycle from preclinical to postlaunch. The focus of each review is to evaluate the case for a new drug or class in outcome terms in specific indications and patient groups

Submit your manuscript here: http://www.dovepress.com/core-evidence-journal

\section{Dovepress}

The manuscript management system is completely online and includes a very quick and fair peer-review system, which is all easy to use. Visit http://www.dovepress.com/testimonials.php to read real quotes from published authors. 